\title{
A Maintainability Growth Model of Complicated Weapon System Based on Virene Algorithm
}

\author{
Zhaojun $\mathrm{WU}^{1, \mathrm{a}}$, Xiangqun $\mathrm{LV}^{1}$, Ming $\mathrm{AN}^{2}$, Dimin $\mathrm{WU}^{1}$ \\ ${ }^{1}$ Wuhan Mechanical Technology College, Wuhan, 430075, China \\ ${ }^{2}$ PLA 65583 Unit, Liaoyang, 111000, China \\ aemail: semicopy@163.com
}

Keywords: Complicated Weapon System; Maintainability Growth; Gompertz Model; Virene Algorithm

\begin{abstract}
Research on maintainability growth is an important research direction for the improvement in the equipment maintainability. A rational maintainability growth model can be utilized to predict the direction and level of maintainability growth. Moreover, it can provide theoretical and decision support for maintainability growth research of complicated weapon system.
\end{abstract}

\section{Introduction}

Maintainability is the ability of a weapon system to maintain and restore the required level, according to the specified repairing procedures and methods under specified conditions and within specified time. Maintainability depends both on the equipment itself, and many external factors which belong to the maintenance support, including the structural design factor, the maintenance support resource, and the maintenance personnel quality. There are many problems, such as, technical difficulties, long time and high cost, during the maintenance of active equipment, especially complicated weapon system. Therefore, improvement or modification of equipment maintenance in the whole life cycle maintenance plan, to achieve sustained growth, and comprehensively improve the level of equipment maintenance, is an urgent need for equipment as soon as possible. Maintainability growth works on the influence factors of active equipment, in order to make the equipment maintenance more convenient. It is a kind of system engineering work, which requires a combination of specific equipment and various constraint conditions to plan.

The definition and process of maintainability growth, together with the rules of evolvement of maintainability, are studied in [1]. The effect of maintainability growth on equipment life cycle cost is illustrated in [2]. Liao et al [3] study trend test of maintainability (test-fix and delay-fix). The Bayes small swatch method is used to estimate the system repair rate [4]. The method is testified in maintainability growth application. Cao et al [5] uses the Gompertz model to estimate the future maintainability of weapon equipment. In this paper, the Gompertz model is utilized to model the maintainability growth of a complicated weapon system, and the model is resolved by Virene algorithm.

\section{Complicated weapon system maintainability growth model}

The connotation of maintainability growth of complex weapon system includes two aspects of the content, i.e., the direction of growth, which is a positive growth or vice growth, and the level of growth, which is to make clear that how much it increase. Consequently, how to measure the direction and extent of the growth, is a real problem in the research of maintainability growth. The usual method of weighing direction and extent of the maintainability increases is contrasting the mean time to repair (MTTR) or the degree of maintenance from beginning to end. The typical problem of this method is that it is difficult to directly and intuitively reflect the general laws of change before and after the maintenance by utilizing MTTR, which is deeply influenced by the repair personnel own quality, and the sample size of repair time, et al. Therefore, developing 
maintainability growth model of weapon equipment especially complicated weapon system, in order to accurately verify or infer the direction and extent of maintainability growth, is a kind of pioneering work, which is significant to improve the maintainability of complex weapon system.

According to the analysis of the concept of maintainability growth and growth process, the following basic models, as shown in Figure 1, need to be established to solve the life cycle stages of maintainability growth.

(1) Maintenance evaluation model. This kind of model is mainly used for solving the prediction, evaluation and expectation of the level of maintenance, in order to make clear the initialization of maintainability growth.

(2) Maintainability defects analysis model. This kind of model is mainly used to classify the maintenance problem, identify the location of defect, find out the reasons, analyze the influence, and put forward the improvement methods.

(3) Growth planning model. This kind of model is mainly used for balancing and optimizing the growth plan, planning an optimal scheme to achieve the desired goals, by considering the object and the method and so on, which has influence on maintainability growth.

(4) Growth validation model. This kind of model is mainly to solve the problem of verification of growth effect, compare the experiment results to the expectations, evaluate the level of growth, and provides a foundation for the next stage of growth.

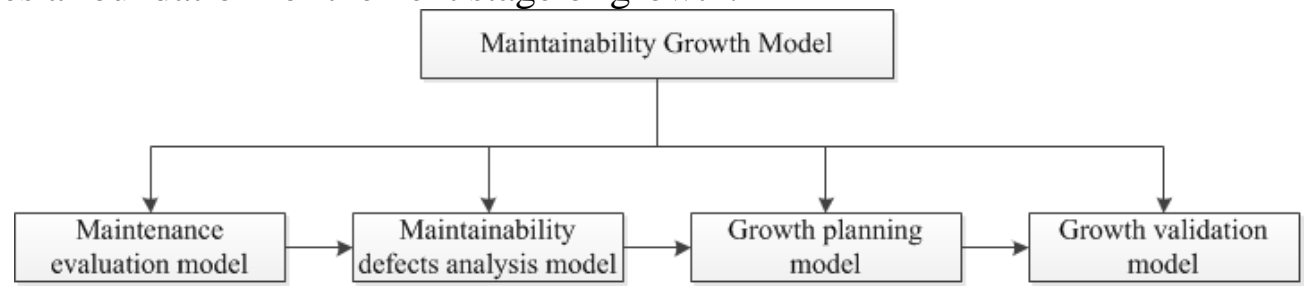

Fig.1. Complicated weapon system maintainability growth model

These models are mutual influence, mutually reinforcing relationship. Maintainability growth analysis can be more scientific, by utilizing the test and data analysis of the model to modify these models continuously. Considering that the complex structure and the numerous sub system and module of large complex weapon system, the basic procedure of the study of complicated weapon system maintainability growth is shown in Figure 2.

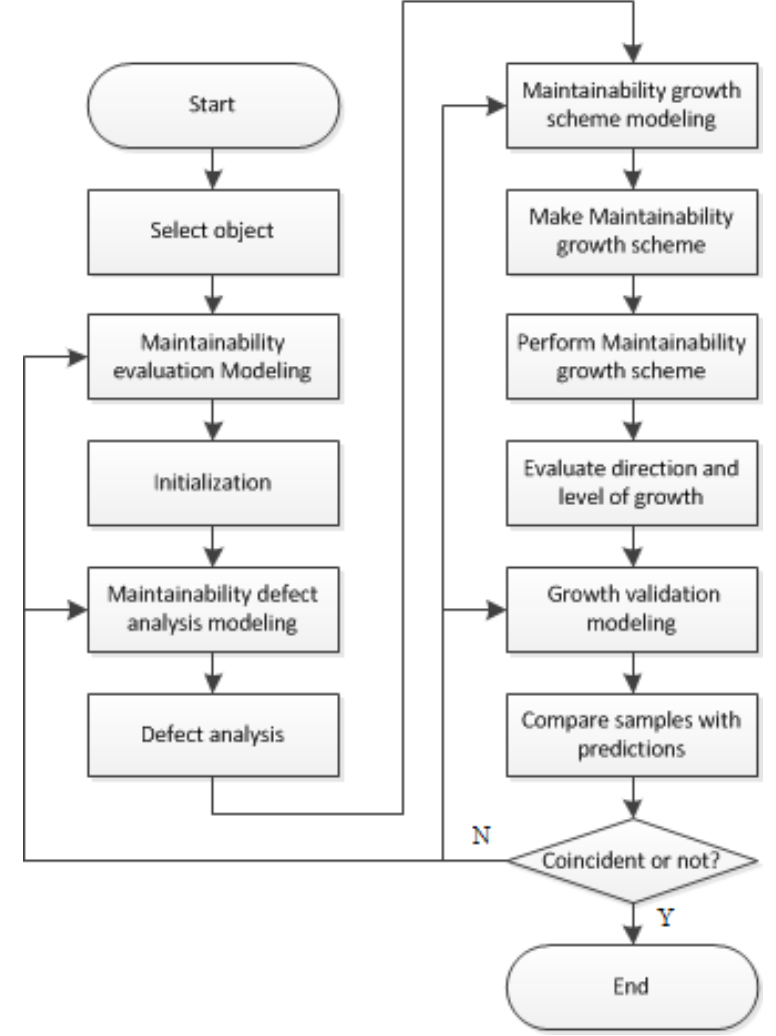

Fig.2. Complicated weapon system maintainability growth research flow chart 


\section{Complicated weapon system maintainability growth based on Virene algorithm}

The original Gompertz model proposed by B.Gompertz is used for the analysis of population growth. In 1968, Gompertz model is used by E.P.Virene to describe the growth curve of reliability [6]. The characteristic of Gompertz model curve is that, it grows slowly at first, and then accelerates gradually until reaches a certain point, after that, the growth rate began to slow down. With respect to the disciplinarian of complicated weapon system maintenance, the Gompertz model based on Virene algorithm can be used to model the maintainability growth of complicated weapon system.

The Gompertz model can be used for preliminary assessment of current and future maintenance equipment maintainability growth model. It is a kind of discrete type. Let the maintainability of equipment at time $\mathrm{t}$ to be $\mathrm{M}(\mathrm{t})$, the Gompertz model can be written as

$$
M(t)=a b^{c^{t}}, 0<a<1,0<b<1,0<c<1
$$

where a represents the upper limit of maintainability growth, ab is the initial maintainability; $b$ is the ratio of initial and final maintainability; c stands for the velocity of maintainability growth.

The Virene algorithm can be utilized in the Gompertz model parameter estimation. Suppose that $\mathrm{n}=3 \mathrm{~m}$, the observed values are $\left(\mathrm{j}, \mathrm{M}_{\mathrm{j}}\right), \mathrm{j}=0,1,2, \ldots, 3 \mathrm{~m}-1$. With Equation (1), we have

$\ln M_{j}=\ln a+c^{\prime} \ln b, \quad j=0,1,2, \ldots, 3 \mathrm{~m}-1$

divide $3 \mathrm{~m}$ observations into 3 average groups, define that

$$
\begin{gathered}
S_{1}=\sum_{j=0}^{m-1} \ln M_{j}=m \ln a+\ln b \sum_{j=0}^{m-1} c^{j} \\
S_{2}=\sum_{j=m}^{2 m-1} \ln M_{j}=m \ln a+\ln b \sum_{j=m}^{2 m-1} c^{j} \\
S_{3}=\sum_{j=2 m}^{3 m-1} \ln M_{j}=m \ln a+\ln b \sum_{j=2 m}^{3 m-1} c^{j}
\end{gathered}
$$

It follows that

$$
\frac{S_{3}-S_{2}}{S_{2}-S_{1}}=\frac{\sum_{j=2 m}^{3 m-1} c^{j}-\sum_{j=-1}^{2 m-1} c^{j}}{\sum_{j=m}^{2 m-1} c^{j}-\sum_{j=0}^{m-1} c^{j}}=c^{m}
$$

Therefore

$$
\hat{c}=\left[\frac{S_{3}-S_{2}}{S_{2}-S_{1}}\right]^{\frac{1}{m}}
$$

Substitution of Equation (7) into Equations (3) and (4), we have

$$
\widehat{a}=\exp \left\{\frac{1}{m}\left[S_{1}+\frac{S_{2}-S_{1}}{1-c^{m}}\right]\right\}, \hat{b}=\exp \left\{\frac{\left(S_{2}-S_{1}\right)(c-1)}{\left(1-c^{m}\right)^{2}}\right\}
$$

\section{Numerical Experiment}

The maintainability growth of some new rocket weapon system's pitching and balancing machine has been studied, and the performance was divided into 9 stages. The maintainability samples of the former 6 stages are listed in Table 1. Modification has been made after each test, in order to enhance the level of maintainability.

The samples are divided into 3 groups, and it follows that

$S_{1}=\ln M_{0} M_{1}=-0.9598, \quad S_{2}=\ln M_{2} M_{3}=-0.5677, \quad S_{3}=\ln M_{4} M_{5}=-0.3583$

Then we have

$\hat{a}=93.7987, \hat{b}=0.5999, \hat{c}=0.7266$

And the Gompertz model can be formulated as. 


$$
\widehat{M}^{(1)}(t)=93.7987 \times 0.5999^{0.7266 t}
$$

Similarly, the predictions of $\hat{M}_{j}^{(1)}$ at other stages can be calculated, so do the error between the samples and the predictions, which are also listed in Table 1.

It can be seen from Table 1 that, at the former 6 stages, the error between the predictions calculated by the Gompertz model and the samples are less than $0.1 \%$, which means that the prediction is coincident with real value, and the Gompertz model can be utilized as a rational prediction model of the maintainability growth. According to the Gompertz model, the maintainability of the pitching and balancing machine can arrive at $90.6111 \%$ after 9 stages of modification.

Tab.1. A new rocket weapon system's pitching and balancing machine maintainability growth experiment data.

\begin{tabular}{c|c|c|c}
\hline Stage $\mathrm{j}$ & $M_{j}(\%)$ & $\hat{M}_{j}^{(1)}(\%)$ & $\hat{M}_{j}^{(1)}-M_{j}(\%)$ \\
\hline 0 & 57.9 & 57.9663 & 0.0663 \\
1 & 65.6 & 65.7475 & 0.1525 \\
2 & 71.5 & 71.4251 & -0.0251 \\
3 & 77.7 & 77.7766 & -0.0749 \\
4 & 81.7 & 81.8425 & 0.1425 \\
5 & 85.2 & 85.2513 & 0.0513 \\
6 & & 86.9175 & \\
7 & & 89.3777 & \\
8 & & 90.6111 & \\
\hline
\end{tabular}

\section{Conclusion}

The Gompertz model based on Virene algorithm is utilized to study the maintainability growth problem of weapon equipment. A series of key techniques of quantitative analysis and prediction of maintainability growth degree are resolved. Numerical experiment shows that the application of the proposed model to evaluate and predict the equipment maintainability growth can improve the analysis efficiency and save the experiment cost. It is an effective analysis method.

\section{References}

[1] Liu Hui, Hao Jian-ping. Study of Maintainability Growth and its Process [J]. Journal of Ordnance Engineering College, 2009, 21(2): 1-5.

[2] He Yu-chun, Jin Jia-shan. Discussion on the influence of maintainability growth of the equipment life cycle cost [C]. Seventh National Conference, LCC Committee of China Association of Plant Management, 2007.

[3] Liao Wu, Chen Yun-xiang. Study on Trend Test of Maintainability Growth [J]. Fire Control and Command Control, 2009, 34(9): 118-121.

[4] Wu Xi, Xu Da, Shao Si-jie, Cao Yan-hua. Research On Maintainability Growth Model of Tank Weapon System Based on the Bayes Method [J]. Aerospace Control, 2010, 28(3): 81-82.

[5] Cao Yan-hua, Guo Jin-mao, Xu Da. Research on Gompertz Model of Equipment Maintainability Growth [J]. Journal of Academy of Armored Force Engineering, 2007, 21(4): 24-27.

[6] Lindley R Higgins. Maintaince Engineering Handbook (6th Edition) [M]. New York: McGraw-Hill, 2002. 